\title{
Disseminated intravascular coagulation observed following treatment with gemtuzumab ozogamicin for relapsed/refractory acute promyelocytic leukemia
}

\author{
YOSHIKO AZUMA, AYA NAKAYA, MASAAKI HOTTA, SHINYA FUJITA, YUKIE TSUBOKURA, \\ HIDEAKI YOSHIMURA, ATSUSHI SATAKE, KAZUYOSHI ISHII, TOMOKI ITO and SHOSAKU NOMURA
}

The First Department of Internal Medicine, Kansai Medical University, Hirakata, Osaka 573-1010, Japan

Received August 24, 2015; Accepted April 7, 2016

DOI: $10.3892 / \mathrm{mco} .2016 .864$

\begin{abstract}
Gemtuzumab ozogamicin (GO) is a recombinant humanized immunoglobulin G4 anti-cluster of differentiation (CD)33 monoclonal antibody conjugated to N-acetyl- $\gamma$ calicheamicin dimethylhydrazide, a naturally potent antibiotic. It has been introduced for the treatment of acute promyelocytic leukemia (APL), since large quantities of CD33 are commonly expressed on the surface of APL cells. The present study reported two cases with prominent disseminated intravascular coagulation (DIC), which was transiently observed following treatment with GO with relapsed/refractory APL. Very limited information exists regarding DIC occurring following GO, and its mechanism remains to be elucidated. In the present study, recombinant human soluble thrombomodulin was used for DIC treatment, and the patients recovered promptly. Since DIC is the most serious adverse event associated with GO treatment, elucidation of its mechanism and establishment of a treatment strategy are warranted.
\end{abstract}

\section{Introduction}

Gemtuzumab ozogamicin (GO) is a recombinant humanized immunoglobulin (Ig)G4 anti-cluster of differentiation (CD)33 monoclonal antibody conjugated to N-acetyl- $\gamma$ calicheamicin dimethylhydrazide, a naturally potent antibiotic. It has been introduced for the treatment of acute promyelocytic leukemia (APL), since large quantities of CD33 are commonly expressed on the surface of APL cells. Although several previous studies have reported successful results for the use of GO as APL therapy (1-8), no large clinical studies of GO for APL treatment have been performed. APL is

Correspondence to: Dr Aya Nakaya, The First Department of Internal Medicine, Kansai Medical University, 2-5-1 Shin-machi, Hirakata, Osaka 573-1010, Japan

E-mail: nakaya1016@yahoo.co.jp

Key words: gemtuzumab ozogamicin, acute promyelocytic leukemia, disseminated intravascular coagulation characterized by fibrinolytic-type disseminated intravascular coagulation (DIC). The present study reported two patients who developed DIC following treatment with GO, although their coagulation profiles revealed no presence of DIC prior to the treatment. Since prominent DIC was transiently observed following treatment with GO, it may be an adverse event caused by GO. Very limited information exists regarding DIC occurring following treatment with GO (9), and its mechanism remains unclear. The present study used recombinant human soluble thrombomodulin (rTM) for the treatment of DIC, although rTM in combination with GO has not been previously reported, to the best of our knowledge. The present study reported on these two patients with relapsed/refractory APL who exhibited DIC following treatment with $\mathrm{GO}$, receiving rTM therapy for DIC.

\section{Case reports}

Case 1. An 85-year-old man presented with pancytopenia in August 2011 at the First Department of Internal Medicine, Kansai Medical University (Osaka, Japan). A chromosome analysis revealed $46, \mathrm{XY}, \mathrm{t}(15 ; 17)$ (q22;21), and APL was diagnosed. Treatment with all-trans retinoic acid (ATRA) and chemotherapy, including idarubicin (IDA), was administered, and hematological complete remission (CR) was attained in November 2011. The patient received maintenance chemotherapy, according to the PETHEMA LPA 2005 regimen (10), and achieved molecular CR in December 2011. The patient continued to receive ATRA until he developed interstitial pneumonia in April 2012. In October 2012, the patient had a molecular relapse and restarted ATRA, however, the disease was refractory. In December 2012, the patient received arsenic trioxide (ATO) and achieved a second CR. In October 2013, the patient had a third molecular relapse. A bone marrow smear revealed $1.8 \%$ APL cells. The mRNA of promyelocytic leukemia (PML)/retinoic acid receptor (RAR) $\alpha$ was detected by reverse transcription-quantitative polymerase chain reaction (RT-qPCR; $1.8 \times 10^{4}$ copies $/ \mu \mathrm{g}$ ). The results of laboratory tests were as follows: Erythrocyte count, $3.93 \times 10^{12} / 1$; platelet count, $15.1 \times 10^{9} / 1$; leukocyte count, $5.9 \times 10^{9} / 1$ (the leukocytes included $0 \%$ promyelocytes, $4.5 \%$ monocytes, $45.5 \%$ neutrophils and $34.5 \%$ lymphocytes). GO 


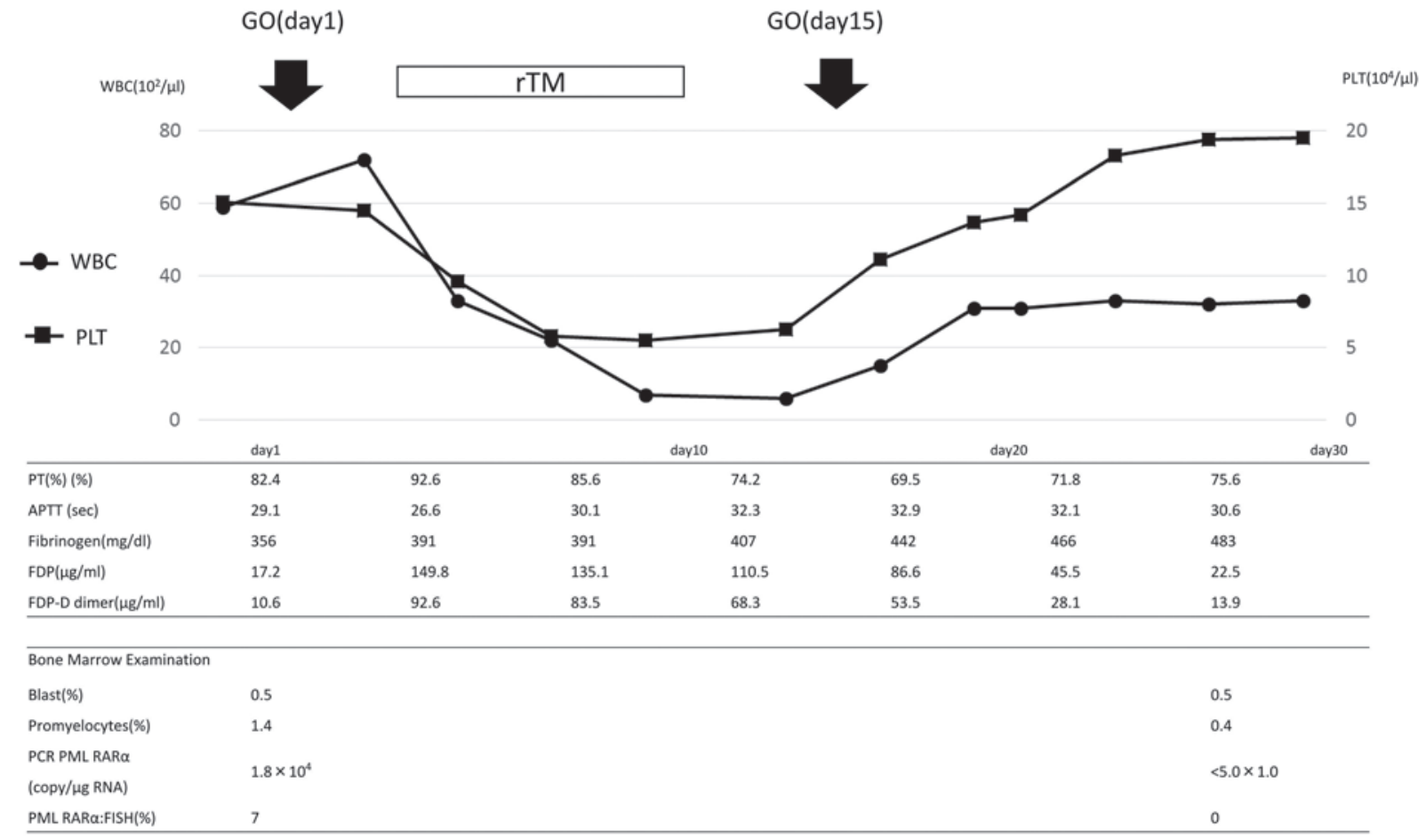

Figure 1. Clinical course of the patient in case 1. GO, gemtuzumab ozogamicin; PT, prothrombin time; WBC, white blood cell count; PLT, platelets; rTM, recombinant human soluble thrombomodulin; APTT, activated partial thromboplastin time; FDP, fibrin degradation product; PCR, polymerase chain reaction; PML, promyelocytic leukemia; RAR, retinoic acid receptor; FISH, fluorescent in situ hybridization.

$\left(9 \mathrm{mg} / \mathrm{m}^{2}\right)$ was administered on days 1 and 15 . The clinical course is shown in Fig. 1. Prominent DIC was transiently observed following the treatment. On day 4 , the prothrombin time (PT) was $92.6 \%$, the activated partial thromboplastin time (APTT) was $26.6 \mathrm{sec}$, the fibrinogen level was $391 \mathrm{~g} / \mathrm{l}$, the fibrin degradation product (FDP) level was $149.8 \mu \mathrm{g} / \mathrm{ml}$ and the fibrin degradation product D-dimer (FDP-DD) level was elevated to $92.6 \mu \mathrm{g} / \mathrm{ml}$. Treatment with rTM was initiated. During this treatment, the FDP and FDP-DD levels gradually decreased. A reduction in the level of fibrinogen was not observed. GO was administered again on day 15 . This caused a slight increase in FDP-DD levels, which promptly decreased. Molecular CR was confirmed on day 41 .

Case 2. An 80-year-old man was diagnosed with APL in 2004 at the First Department of Internal Medicine, Kansai Medical University. The patient was administered treatment with ATRA plus IDA, and CR was attained. The patient relapsed in June 2012. A second treatment with ATRA was administered and the patient exhibited a second CR. A second relapse occurred and the patient received ATO, however, this was subsequently discontinued due to QT interval prolongation. Tamibarotene was started, however, the disease was resistant. A bone marrow smear revealed 22\% APL cells. The mRNA of PML/RAR $\alpha$ was detected by RT-qPCR $\left(8.3 \times 10^{4}\right.$ copies $\left./ \mu \mathrm{g}\right)$. The results of laboratory tests were as follows: Erythrocyte count, $2.43 \times 10^{12} / 1$; platelet count, $10.3 \times 10^{9} / 1$; leukocyte count, $9.0 \times 10^{9} / 1$ (the leukocytes included $0 \%$ promyelocytes, $1 \%$ monocytes, $55 \%$ neutrophils and $43 \%$ lymphocytes).

GO $\left(9 \mathrm{mg} / \mathrm{m}^{2}\right)$ was administered on day 1 . The clinical course is shown in Fig. 2. Although the coagulation profile revealed no presence of DIC prior to the treatment with GO, prominent DIC was transiently observed following the treatment. On day 4, the PT was $77.6 \%$, the APTT was $29.4 \mathrm{sec}$, the fibrinogen level was $350 \mathrm{~g} / \mathrm{l}$, the FDP level was $176.4 \mu \mathrm{g} / \mathrm{ml}$ and the FDP-DD level was elevated to $109 \mu \mathrm{g} / \mathrm{ml}$. rTM treatment was initiated, and the FDP and FDP-DD level gradually decreased. On day 9, the patient acquired a severe infection, which transiently increased the FDP-DD level. Following recovery from the infection, the FDP-DD level returned to normal. During the treatment course, reduction of the fibrinogen level was not observed. Due to the infection, the patient was unable to receive GO on day 15. Molecular CR was confirmed on day 24 .

\section{Discussion}

$\mathrm{GO}$ is a recombinant humanized IgG4 anti-CD33 monoclonal antibody conjugated to $\mathrm{N}$-acetyl $-\gamma$ calicheamicin dimethylhydrazide, a naturally potent antibiotic. It has been introduced for the treatment of APL (1-8), although its efficacy in ATRAand chemotherapy-resistant fully-relapsed APL remains to be elucidated. Certain basic reasons may explain the effectiveness of GO for the treatment of APL. Firstly, large quantities of CD33 are commonly expressed on the surface of APL cells. Secondly, the levels of P-glycoprotein (a multidrug-resistant glycoprotein) are lower on the surface of APL cells compared with on the surface of acute myeloid leukemia (AML) cells. Thirdly, APL cells are highly sensitive to free calicheamicin, an antitumor antibiotic $(2,3)$.

Several previous studies have hypothesized the efficacy of GO for APL $(1,5)$. In a previous study performed at the MD Anderson Cancer Center (Houston, TX, USA), the CR 


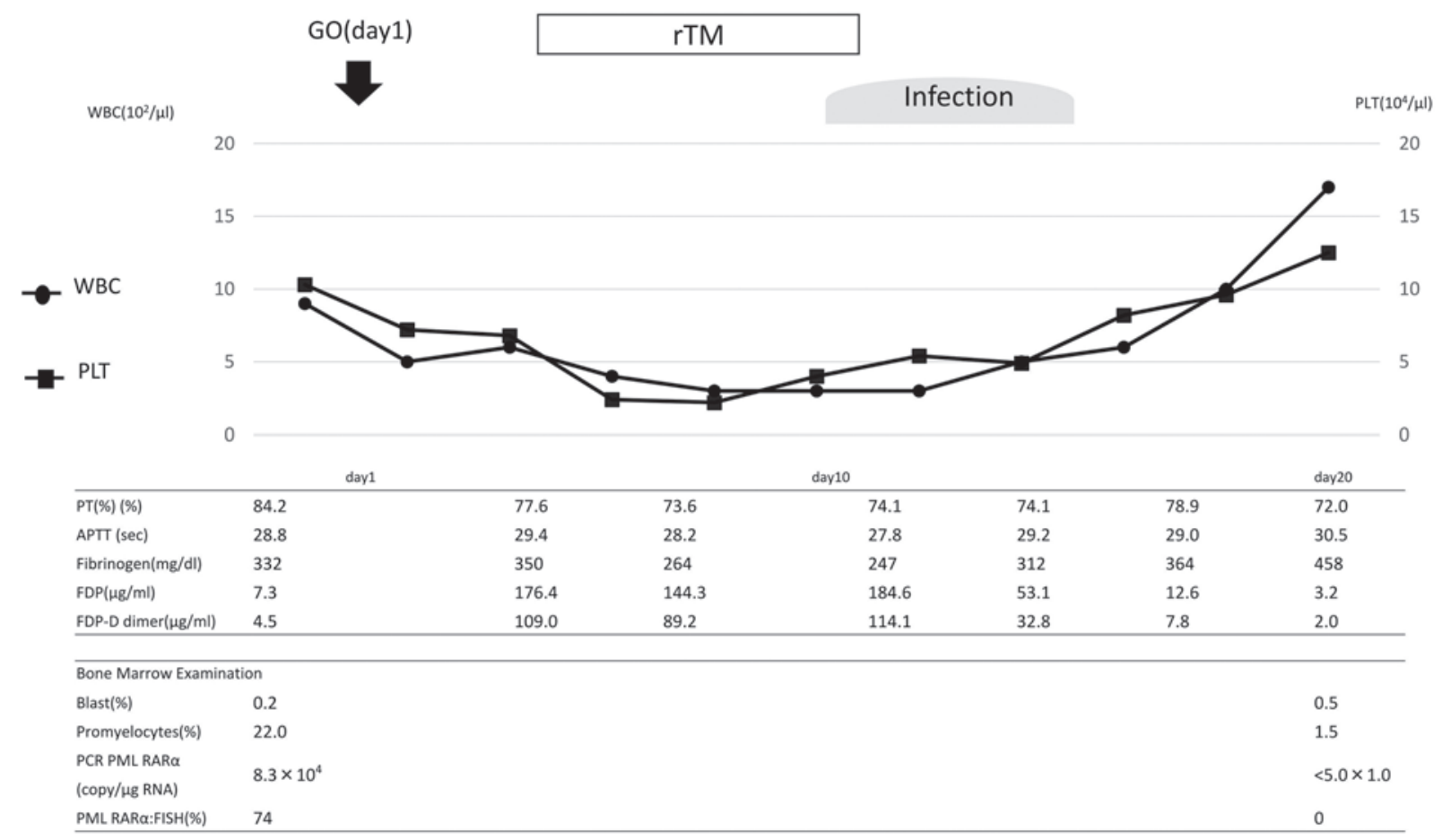

Figure 2. Clinical course of the patient in case 2. GO, gemtuzumab ozogamicin; PT, prothrombin time; WBC, white blood cell count; PLT, platelets; rTM, recombinant human soluble thrombomodulin; APTT, activated partial thromboplastin time; FDP, fibrin degradation product; PCR, polymerase chain reaction; PML, promyelocytic leukemia; RAR, retinoic acid receptor; FISH, fluorescent in situ hybridization.

rate was $81 \%$ in high-risk patients who received GO (5). The combination of ATRA and ATO plus GO is now being evaluated in a North American Intergroup APL trial for high-risk APL. Italian investigators noted that early treatment of molecular relapse of APL with single-agent GO resulted in longer survival compared with the initiation of treatment at hematological relapse (1).

The relative rarity of APL, particularly molecular relapse of APL, makes a randomized study to confirm these findings infeasible. The SWOG106 study, comprising a trial of GO with cytarabine plus anthracycline in relapsed AML, found no improvement in event-free survival, disease-free survival or overall survival (11). These results prompted Pfizer to voluntarily withdraw GO from the market in the United States in June 2010, prior to the results of other randomized trials being made available.

A post-marketing study of GO in Japan reported that the frequencies of adverse events were myelosuppression (67\%), infusion reaction (47\%), liver dysfunction (43\%), infection (35\%), bleeding (17\%) and DIC (7\%). Prominent DIC following treatment with GO was reported in two previous cases (9). In that previous study, the investigators assumed that GO may increase the frequency and severity of DIC due to APL cells collapsing rapidly. However, the frequency of DIC in the post-marketing surveillance was $7 \%$, meaning that DIC does not necessarily occur in all cases. If DIC arose through the collapse of APL cells, it may be proportional to the quantity of APL cells. In the present study, case 1 exhibited a molecular relapse, however, the number of APL cells was so small that it could not be counted in the bone marrow smear and blood tests. Therefore, the quantity of tumor cells must have been small, however, DIC still occurred. By contrast, following the treatment with GO on day 15, DIC did not occur. From this clinical course, it can be assumed that DIC must have occurred through a mechanism other than the quantity of APL cells. The present study hypothesized that GO may damage vascular endothelial cells and cause DIC, since GO is said to be associated with the development of sinusoidal obstruction. The mechanism of sinusoidal obstruction caused by GO remains unclear, however, it may be similar to DIC. Since the initial onset of DIC and sinusoidal obstruction is vascular endothelial damage, it was assumed that rTM may prevent DIC from occurring in the administration of 15 days of GO. The present study initiated rTM on day 4, which was soon after DIC occurred and continued it until day 11, then administered GO on day 15 . The present study hypothesized that the effects of rTM remained on day 15 and assisted with the treatment of endothelial damage, therefore, the increase of FDP-DD was less than on day 1 .

APL is characterized by fibrinolytic-type DIC, which is characterized by increasing levels of FDP-DD and decreasing levels of fibrinogen. rTM was approved for treatment of DIC in Japan in 2008. Previously, heparin and gabexate mesilate were used prophylactically prior to the treatment with GO (9). In the present study, rTM was used for the first time, to the best of our knowledge, for DIC triggered by GO. Although a report indicated that rTM was effective in the treatment with ATRA or ATO for DIC (12), its usefulness for treatment of APL has not been confirmed in larger studies. No bleeding-associated mortality was noted during induction chemotherapy in APL patients who received rTM (13). Notably, severe hemorrhage requiring red blood cell transfusion at the time of diagnosis of APL was reduced following the initiation of rTM (14). Use of rTM in combination with ATO for relapse of APL 
promptly improved DIC without any adverse effects in one previous case report (15). An in vitro study demonstrated that exposure of APL cells to rTM significantly downregulated the level of annexin II, resulting in a decrease in plasmin production (16). However, the mechanism remains to be elucidated in vivo.

rTM was stopped after 7 days, since its safety for use over 7 days remains to be determined. In the present case, following treatment with rTM, the efficacy of rTM remained and FDP-DD kept decreasing.

Each of the cases in the present study achieved early molecular CR following treatment with GO. Therefore, GO may be a promising agent for the management of APL and may become one of the treatment options for recurrent APL in elderly patients. Since DIC is the most serious adverse event of GO treatment, elucidation of its mechanism and establishment of a treatment strategy are warranted.

\section{References}

1. Lo-Coco F, Cimino G, Breccia M, Noguera NI, Diverio D, Finolezzi E, Pogliani EM, Di Bona E, Micalizzi C, Kropp M, et al: Gemtuzumab ozogamicin (Mylotarg) as a single agent for molecularly relapsed acute promyelocytic leukemia. Blood 104: 1995-1999, 2004.

2. Takeshita A, Shinjo K, Naito K, Matsui H, Sahara N, Shigeno K, Horii T, Shirai N, Maekawa M, Ohnishi K, et al: Efficacy of gemtuzumab ozogamicin on ATRA- and arsenic-resistant acute promyelocytic leukemia (APL) cells. Leukemia 19: 1306-1311, 2005.

3. Aribi A, Kantarjian HM, Estey EH, Koller CA, Thomas DA, Kornblau SM, Faderl SH, Laddie NM, Garcia-Manero G and Cortes JE: Combination therapy with arsenic trioxide, all-trans retinoic acid, and gemtuzumab ozogamicin in recurrent acute promyelocytic leukemia. Cancer 109: 1355-1359, 2007.

4. Sanz MA, Montesinos P, Rayón C, Holowiecka A, de la Serna J, Milone G, de Lisa E, Brunet S, Rubio V, Ribera JM, et al: Risk-adapted treatment of acute promyelocytic leukemia based on all-trans retinoic acid and anthracycline with addition of cytarabine in consolidation therapy for high-risk patients: Further improvements in treatment outcome. Blood 115: 5137-5146, 2010.

5. Ravandi F, Estey E, Jones D, Faderl S, O'Brien S, Fiorentino J, Pierce S, Blamble D, Estrov Z, Wierda W, et al: Effective treatment of acute promyelocytic leukemia with all-trans-retinoic acid, arsenic trioxide and gemtuzumab ozogamicin. J Clin Oncol 27: 504-510, 2009
6. Ravandi F, Estey EH, Appelbaum FR, Lo-Coco F, Schiffer CA, Larson RA, Burnett AK and Kantarjian HM: Gemtuzumab ozogamicin: Time to resurrect? J Clin Oncol 30: 3921-3923, 2012 .

7. Lo-Coco F, Latagliata R and Breccia M: Management of acute promyelocytic leukemia in the elderly. Mediterr J Hematol Infect Dis 5: e2013045, 2013.

8. Takeshita A: Efficacy and resistance of gemtuzumab ozogamicin for acute myeloid leukemia. Int J Hematol 97: 703-716, 2013.

9. Takeshita A, Shinjo K, Naito K, Matsui H, Sahara N, Shigeno K, Suzumura T, Horii T, Shirai N, Maekawa M, et al: Two patients with all-trans retinoic acid-resistant acute promyelocytic leukemia treated successfully with gemtuzumab ozogamicin as a single agent. Int J Hematol 82: 445-448, 2005.

10. Sanz MA, Martín G, González M, León A, Rayón C, Rivas C, Colomer D, Amutio E, Capote FJ, Milone GA, et al: Risk-adapted treatment of acute promyelocytic leukemia with all-trans-retinoic acid and anthracycline monochemotherapy: A multicenter study by the PETHEMA group. Blood 103: 1237-1243, 2004.

11. Petersdorf SH, Kopecky KJ, Slovak M, Willman C, Nevill T, Brandwein J, Larson RA, Erba HP, Stiff PJ, Stuart RK, Walter RB, Tallman MS, Stenke L and Appelbaum FR. A phase 3 study of gemtuzumab ozogamicin during induction and postconsolidation therapy in younger patients with acute myeloid leukemia. Blood 121: 4854-4860, 2013.

12. Ikezoe T: Pathogenesis of disseminated intravascular coagulation in patients with acute promyelocytic leukemia, and its treatment using recombinant human soluble thrombomodulin. Int J Hematol 100: 27-37, 2014

13. Ikezoe T, Takeuchi A, Isaka M, Arakawa Y, Iwabu N, Kin T, Anabuki K, Sakai M, Taniguchi A, Togitani K and Yokoyama A: Recombinant human soluble thrombomodulin safely and effectively rescues acute promyelocytic leukemia patients from disseminated intravascular coagulation. Leuk Res 36: 1398-1402, 2012.

14. Kawano N, Kuriyama T, Yoshida S, Yamashita K, Ochiai H, Nakazaki S, Tasaki A and Ueda A: Clinical features and treatment outcomes of six patients with disseminated intravascular coagulation resulting from acute promyelocytic leukemia and treated with recombinant human soluble thrombomodulin at a single institution. Intern Med 52: 55-62, 2013.

15. Shindo M, Ikuta K, Addo L, Ito S, Fujiya M, Torimoto Y and Kohgo Y: Successful control of disseminated intravascular coagulation by recombinant thrombomodulin during arsenic trioxide treatment in relapsed patient with acute promyelocytic leukemia. Case Rep Hematol 2012: 908196, 2012.

16. Ikezoe T, Yang J, Nishioka C, Isaka M, Iwabu N, Sakai M, Taniguchi A, Honda G and Yokoyama A: Thrombomodulin enhances the antifibrinolytic and antileukemic effects of all-trans retinoic acid in acute promyelocytic leukemia cells. Exp Hematol 40: 457-465, 2012. 